\title{
BUILDING 3D SURFACES OF LAND STORAGE VERTICAL CYLINDRICAL STEEL TANK USING BICUBIC SPLINE INTERPOLATION
}

\author{
Kostyantyn BURAK ${ }^{1}$, Vitaliy KOVTUN ${ }^{1 *}$, Mary NYCHVYD ${ }^{2}$ \\ ${ }^{1}$ Ivano-Frankivsk National Technical University of Oil and Gas, Ivano-Frankivsk, Ukraine \\ ${ }^{2}$ Uzhgorod National University, Uzhgorod, Ukraine
}

Received 05 November 2018; accepted 03 June 2019

\begin{abstract}
The purpose of this work is to increase the accuracy, quality and information content of geodetic surveys of vertical steel tanks by using modern geodetic equipment and creating algorithms for data processing of these observations. Method. In order to increase the information content of data for straightening, it is proposed to calculate the geometric parameters of vertical steel tanks not only in places where data are directly obtained through instrumental observations, but also at any point of the 3D surface of the tank. The paper describes an algorithm for creating a 3D surface of a tank by bicubic spline interpolation (BSI). Results on the basis of the conducted research, it was established that the developed algorithm could be used and the 3D-surface spatial coordinates were determined. The method of determining the geometric parameters of vertical steel tanks by using BSI is improved.

Scientific novelty and practical significance. Bicubic spline interpolation (BSI) was used for the first time. It greatly increases the accuracy and informality of the results of the control. The practical significance is confirmed by the control of the geometric parameters of a vertical cylindrical steel tank with a nominal capacity of $75.000 \mathrm{~m}^{3}$ with a floating roof and a double wall of the LODS "Brody" company.
\end{abstract}

Keywords: bicubic spline interpolation, 3D surface, vertical steel tank, geodetic control.

\section{Introduction}

The economic development and welfare of any country in the world largely depends on the security needs of the industry by hydrocarbon raw energy. It does not matter that these energy sources are of their own production or are imported from other countries to the places of their processing, they should be transported by some type of transport. The most economically and technically beneficial for the transportation of oil and oil products among all types of transport is pipeline transport. Ukraine needs about 40 million tons of oil annually. Real annual production at its own fields is $3-4$ million tons. Therefore, the remaining needs, almost 90\% of "black gold", we are forced to get from Russia. Such a monopoly is extremely disadvantageous for Ukraine. For this purpose, to meet the requirements of Ukrainian industry in alternative sources of oil supplies, the Odesa-Brody oil pipeline and the "Pivdennyy" sea oil terminal were built. The oil depots and fuel depots have an important role in the oil supply system. The tanks are important engineering structures; they are destined for receiving, storing, dispensing, and accounting the oil and oil products. Metal tanks are among the important welded constructions, used in difficult operating conditions. The presence in tanks of hard welded joints and the reduction of the plastic properties of the metal at negative temperatures causes significant internal stresses and creates conditions that exclude the possibility of their redistribution. These and a number of other reasons, such as uneven precipitation, corrosion, reduce the operational reliability of the tank, and, sometimes, leading to its destruction. Periodic inspection and integrated defectoscopy allow timely identify a flaws, that were made during the manufacture and construction of tanks, as well as those that appeared during operation. To improve the reliability and term of working efficiency of tanks without sacrificing the current level of oil loading, it is necessary to carry out the engineering inspection (expert examination) of the technical condition of all structural elements of tanks in time and in accordance with regulatory documents and eliminate the identified defects. The problem of reliability and operability of equipment and facilities

*Corresponding author. E-mail: rjdney@gmail.com 
of the main oil pipeline is very important in the field of transport and storage of crude oil and oil products. If the equipment is reliable, there will be less downtime in the work of transporting oil and oil products, accidents with oil spillage and other consequences, harmful to the enterprise and the environment.

Tanks, which are in operation, are subject to technical diagnostics (expert examination) as objects of increased danger (DSTU-NBA.3.1-10:2008 FAT, 2008). According to (SUC 60.3.31570412:2010, 2010), expert examination (technical diagnostics) includes, in addition to defectoscopy, the instrumental geodetic measurements such as leveling the bottom and the roof cover of the tank, determining the deviation of the tank walls from the vertical. Regulatory documents that regulate these works describe the techniques based on the use of outdated tools: carriages, plumb, metal rulers and theodolites. Modern methods of measurement, using electronic total stations and laser scanning (Komissarov, Seredovich, \& Ivanova, 2006), are investigated in the works of professor A. Samoilenko. Its results are presented in (Larichev, Lesonen, Maksimov, Podgyachev, \& Derov, 2010). These methods provide more objective and accurate information about the geometry of the structure. In order to maximize the use of this information, to automate the processes and processing, we carried out the research, using the BSI method to create a 3D surface of vertical steel tank (further VST).

The use of electronic devices such as electronic tachometers or levels, ground-based laser scanners (Samoilenko \& Zaets, 2007; Seredovich \& Ivanov, 2006), significantly reduces the time and increases the order of measurement accuracy. One of the drawbacks, in our opinion, is not full use of the available information, that is, the object of diametral deviations from the verticality of the tank wall is determined only at the observation points, and the height of the tank bottom is determined only on four diagonals, according to the requirements of the current instruction (DSTU-NBA.3.1-10:2008 FAT, 2008). Such sampling control cannot provide a complete picture of the technical condition.

In order to obtain data to estimate the stressed-deformed state at any point in the tank, we propose to create a 3D model, based on the use of bicubic spline interpolation, which meets the accuracy requirements (Burak, Kovtun, Levitskiy, \& Nychvyd, 2014).

\section{Method}

In accordance with regulatory requirements (DSTUNBA.3.1-10:2008 FAT, 2008; SUC 60.3.31570412:2010, 2010; GOST 8.570-2000, 2000), tanks should undergo a complete calibration not less than 10 years with a tank life of up to 20 years and every 8 years when operating for more than 20 years. Therefore, in December 2014, the ground vertical cylindrical steel welded tank with a nominal capacity of $75.000 \mathrm{~m}^{3}$ with a floating roof and a double wall of the LODS "Brody" company (see Figure 2) was decommissioned for a full planned survey.

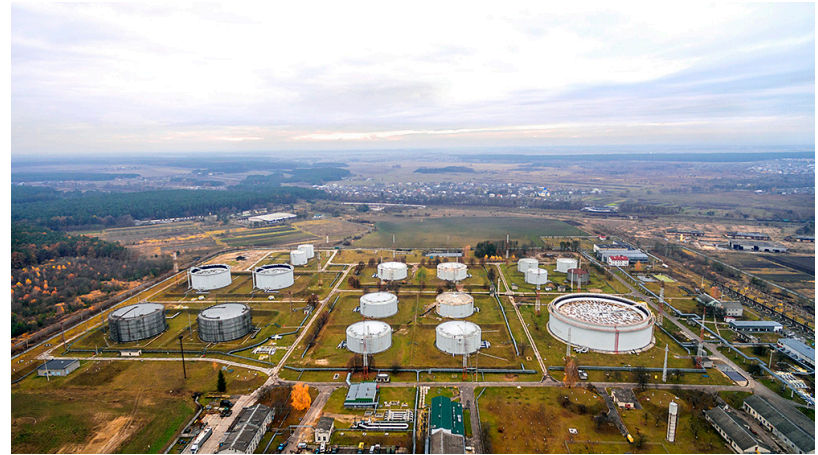

Figure 1. Tank field of LODS "Brody" company

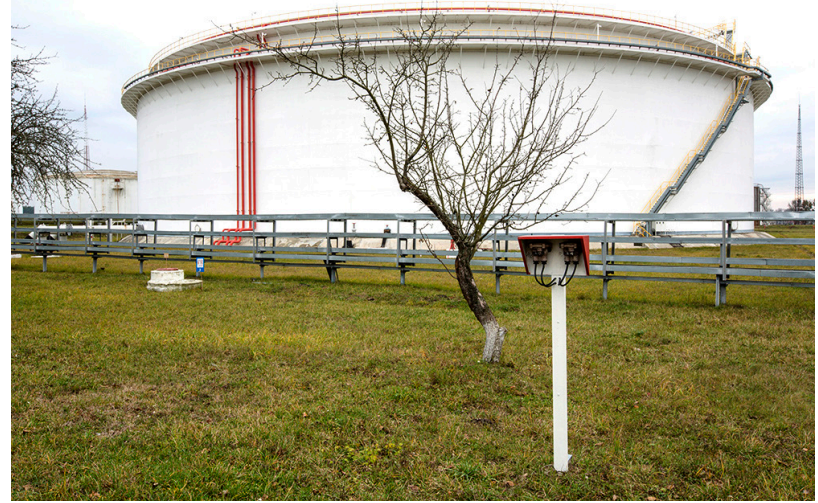

Figure 2. Ground vertical cylindrical steel welded tank with a nominal capacity of $75.000 \mathrm{~m}^{3}$ with a floating roof and a double wall of the LODS "Brody" company

LODS "Brody" company (see Figure 1) is one of the main petroleum pumping stations of the Druzhba oil pipeline and is still the final point of the newly constructed Odesa-Brody oil pipeline system. The LODS "Brody" company has been operating for more than 40 years

Technical characteristics of the study tank:

- type - ground vertical cylindrical welded steel tank with a nominal capacity of $75.000 \mathrm{~m}^{3}$ with a floating roof and a double wall;

- diameter:

- designed internal diameter of the main (internal) tank - $72000 \mathrm{~mm}$;

- actual internal diameter of the main (internal) tank - $71880 \mathrm{~mm}$;

- designed internal diameter of the protective tank $78000 \mathrm{~mm}$;

- actual internal diameter of the protective tank $77890 \mathrm{~mm}$;

- outer diameter of the floating roof $-71540 \mathrm{~mm}$;

- wall height:

- designed height of main (internal) tank $19930 \mathrm{~mm}$;

- actual height of the main (internal) tank $19910 \mathrm{~mm}$;

- designed height of protective tank - $16690 \mathrm{~mm}$;

- actual height of the protective tank - $16170 \mathrm{~mm}$; 
- according to the technical passport on the tank:

- capacity - $75330 \mathrm{~m}^{3}$;

- maximum filling height - $18.5 \mathrm{~m}$;

- the width of the sealing space is $200 \mathrm{~mm}$;

- mass of floating roof with supporting posts 309.69 tons;

- weight of the rolling ladder - 38.54 tons;

- Estimated immersion of a floating roof on water 230-255 mm.

We used the method of determining the deviations of the tank walls from the vertical, which was based on carrying out coordinate measurements of each welding seam which density is suitable for our purpose (Burak, Kovtun, \& Levitskiy, 2015). Depending on the situation around the tank, we chose a geodetic network scheme in the form of a closed triangle, a quadrilateral, a hexagon, or even an octagon. The shortest distance from the points of the geodetic network to the tank generator was from one to four tank heights. It is recommended that the sides of the geodetic network have approximately the same length, and the distances from the points of the geodetic network to the tank wall should be approximately equal. Taking into account (Tarasenko \& Tishchenko, 2009) and (Trevogo, Ilkiv, \& Kukhtar, 2013), measurements of only 4 seam were made by the electronic total station from one station in order to avoid the possibility of a beam slipping or loss of accuracy due to its scattering. Thus, the coordinates of the tank wall along the vertical from the lowest point of the first belt (SUC 60.3.31570412:2010, 2010) at the level of each belt of the tank were obtained.

The measurements were carried out in the conditional coordinate system due to the absence of the planned coordinates of the reference points near the tank. Using certain coordinates of the stations, linear-angular values of the tacheometrical traverse were calculated, which allowed us to compensate it. According to the results of compensation, the tacheometrical traverse (see Figure 3) was measured with a relative accuracy of $1 / 52100$.

After instrumental observations, the coordinates X, Y, and $\mathrm{H}$ were obtained for 278 points in the conventional coordinate system.

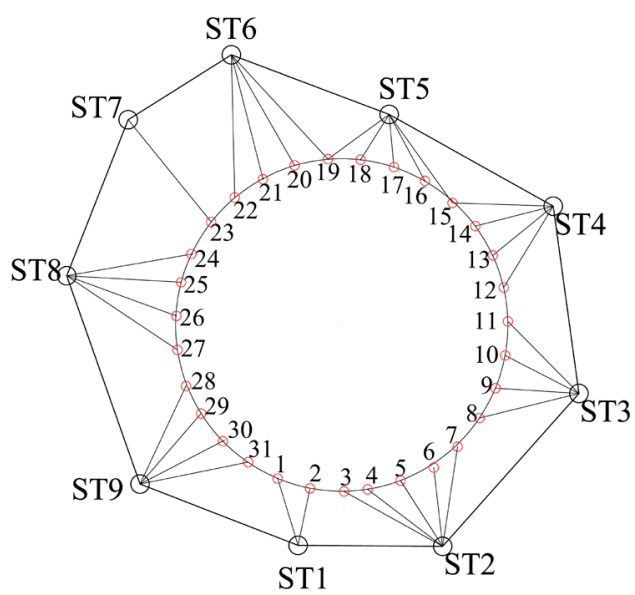

Figure 3. Tacheometric traverse around the tank
To build a 3D surface of the tank, a method for constructing the Hermite bicubic interpolation surface (Alberg, Nilson, \& Walsh, 1972; Shikin \& Plis, 1996) was developed (1).

Let us number the obtained points in a certain order and write in the form of a matrix with elements $M_{i, j}(x, y, z), i=0 . . n, j=0 . . m$, where $n-$ is the number of points along the vertical, the numbering increases from the upper belt to the lower one, and $m$ is the number of points in one belt, the numbering increases counterwise.

To construct an interpolation spline surface with these points, each of $n \times m$ elements are formed by 4 adjacent nodes $M_{i, j}, M_{i+1, j}, M_{i, j+1}, M_{i+1, j+1}$ of the desired surface, we will define by parametric expressions.

$$
\begin{aligned}
& x=\mathbf{r}_{x}^{(i, j)}(s, t) \equiv S^{T}(s) \mathbf{B} \mathbf{M}_{\mathbf{X}}^{(i, j)} \mathbf{B}^{T} T(t), \\
& y=\mathbf{r}_{y}^{(i, j)}(s, t) \equiv S^{T}(s) \mathbf{B} \mathbf{M}_{\mathbf{Y}}^{(i, j)} \mathbf{B}^{T} T(t), \\
& z=\mathbf{r}_{z}^{(i, j)}(s, t) \equiv S^{T}(s) \mathbf{B} \mathbf{M}_{\mathbf{Z}}^{(i, j)} \mathbf{B}^{T} T(t), \\
& s \in[0,1], \quad t \in[0,1],
\end{aligned}
$$

or in vector form:

$$
\begin{aligned}
& \mathbf{r}^{(i, j)}(s, t)=S^{T}(s) \mathbf{B} \mathbf{M}^{(i, j)} \mathbf{B}^{T} T(t), \\
& s \in[0,1], t \in[0,1],
\end{aligned}
$$

where $T(t), S(s)$ - the vectors, the elements of which are formed by degrees of parameters (3)

$$
S^{T}(s)=\left[\begin{array}{llll}
1 & s & s^{2} & s^{3}
\end{array}\right], T(t)=\left[\begin{array}{c}
1 \\
t \\
t^{2} \\
t^{3}
\end{array}\right] ;
$$

B - the base matrix of the spline-surface of Hermite (4)

$$
\mathbf{B}=\left[\begin{array}{cccc}
1 & 0 & 0 & 0 \\
0 & 0 & 1 & 0 \\
-3 & 3 & -2 & -1 \\
2 & -2 & 1 & 1
\end{array}\right]
$$

$\mathbf{M}_{\mathbf{X}}^{(i, j)}, \mathbf{M}_{\mathbf{Y}}^{(i, j)}, \mathbf{M}_{\mathbf{Z}}^{(i, j)}$ and $\mathbf{M}^{(i, j)}$ derivatives and partial derivatives at these points. In particular, for the element, formed by the points $M_{i, j}, M_{i+1, j}, M_{i, j+1}, M_{i+1, j+1}$, matrix $\mathbf{M}_{\mathbf{X}}^{(i, j)}$ will look:

$$
\mathbf{M}_{\mathbf{X}}^{(i, j)}=\left[\begin{array}{cccc}
x_{i, j} & x_{i, j+1} & \partial_{t}^{1} x_{i, j} & \partial_{t}^{1} x_{i, j+1} \\
x_{i+1, j} & x_{i+1, j+1} & \partial_{t}^{1} x_{i+1, j} & \partial_{t}^{1} x_{i+1, j+1} \\
\partial_{s}^{1} x_{i, j} & \partial_{s}^{1} x_{i, j+1} & \partial_{t s}^{2} x_{i, j} & \partial_{t s}^{2} x_{i, j+1} \\
\partial_{s}^{1} x_{i+1, j} & \partial_{s}^{1} x_{i+1, j+1} & \partial_{t s}^{2} x_{i+1, j} & \partial_{t s}^{2} x_{i+1, j+1}
\end{array}\right] .
$$

For the vector function (2) we have respectively:

$$
\mathbf{M}^{(i, j)}=\left[\begin{array}{cccc}
\mathbf{r}_{i, j} & \mathbf{r}_{i, j+1} & \partial_{t}^{1} \mathbf{r}_{i, j} & \partial_{t}^{1} \mathbf{r}_{i, j+1} \\
\mathbf{r}_{i+1, j} & \mathbf{r}_{i+1, j+1} & \partial_{t}^{1} \mathbf{r}_{i+1, j} & \partial_{t}^{1} \mathbf{r}_{i+1, j+1} \\
\partial_{s}^{1} \mathbf{r}_{i, j} & \partial_{s}^{1} \mathbf{r}_{i, j+1} & \partial_{t s}^{2} \mathbf{r}_{i, j} & \partial_{t s}^{2} \mathbf{r}_{i, j+1} \\
\partial_{s}^{1} \mathbf{r}_{i+1, j} & \partial_{s}^{1} \mathbf{r}_{i+1, j+1} & \partial_{t s}^{2} \mathbf{r}_{i+1, j} & \partial_{t s}^{2} \mathbf{r}_{i+1, j+1}
\end{array}\right] .
$$


The symbols $\partial_{t}^{1} \mathbf{r}_{i, j}, \partial_{s}^{1} \mathbf{r}_{i, j}, \partial_{t s}^{2} \mathbf{r}_{i, j}$ meaning the values of the corresponding derivatives of the vector function $\mathbf{r}^{(i, j)}(s, t)$ at $s=0, t=0$ at the points $M_{i, j} \equiv \mathbf{r}_{i, j}$, and $\partial_{t}^{1} x_{i, j}, \partial_{s}^{1} x_{i, j}, \partial_{t s}^{2} x_{i, j}-$ the components on the $\mathrm{x}$-axis, respectively.

The Hermitian form the spline surface for given values $\mathbf{r}_{i, j}, \partial_{t}^{1} \mathbf{r}_{i, j}, \partial_{s}^{1} \mathbf{r}_{i, j}, \partial_{t s}^{2} \mathbf{r}_{i, j}, i=0 . . n, j=0 . . m$ ensures the continuity of the vector function itself, its first and second mixed derivatives at all internal points of the surface. However, in practice, according to the measurement results $M_{i, j} \equiv \mathbf{r}_{i, j}, i=0 . . n, j=0 . . m$, only the coordinates of the points, are usually known, while the values of the derivatives at these points are unknown. In such cases, to determine the unknown quantities at these points $\partial_{t}^{1} \mathbf{r}_{i, j}$, $\partial_{s}^{1} \mathbf{r}_{i, j}, \partial_{t s}^{2} \mathbf{r}_{i, j}$, impose the conditions of continuity of higher derivatives $\partial_{t t}^{2} \mathbf{r}^{(i, j)}(s, t), \partial_{s s}^{2} \mathbf{r}^{(i, j)}(s, t)$ and $\partial_{t s s}^{3} \mathbf{r}^{(i, j)}(s, t)$ or $\partial_{s t t}^{3} \mathbf{r}^{(i, j)}(s, t)$ at the interior points, as well as the boundary conditions at the edges of the surface.

The continuity conditions for the second derivatives at all internal points $M_{i, j}$ (including along the border, except ones on corners) will be:

$\partial_{t t}^{2} \mathbf{r}^{(i, j-1)}(0,1)=\partial_{t t}^{2} \mathbf{r}^{(i, j)}(0,0), i=0 . . n, j=1 . . m-1$,

$\partial_{s s}^{2} \mathbf{r}^{(i-1, j)}(1,0)=\partial_{s s}^{2} \mathbf{r}^{(i, j)}(0,0), i=1 . . n-1, j=0 . . m$.

Calculating the derivatives of expressions (2) and moving the unknown terms to the left, and the known ones to the right, we obtain:

$$
\begin{aligned}
& \partial_{t}^{1} \mathbf{r}_{i, j-1}+4 \partial_{t}^{1} \mathbf{r}_{i, j}+\partial_{t}^{1} \mathbf{r}_{i, j+1}=-3 M_{i, j-1}+3 M_{i, j+1}, \\
& i=0 . . n, j=1 . . m-1 ; \\
& \partial_{s}^{1} \mathbf{r}_{i-1, j}+4 \partial_{s}^{1} \mathbf{r}_{i, j}+\partial_{s}^{1} \mathbf{r}_{i+1, j}=-3 M_{i-1, j}+3 M_{i+1, j}, \\
& i=1 . . n-1, \quad j=0 . . m .
\end{aligned}
$$

For definiteness we assume that a change in the parameter $s$ leads to a change in the vector function $\mathbf{r}^{(i, j)}(s, t)$ along the column (index $i$ ), and variable $t$ - along the row (index $j$ ) of the matrix of nodes $M_{i, j}$. In addition, the location of points on the surface is according to the numbering of the matrix elements $\mathbf{M}^{(i, j)}$ (see Figure 4).

For the construction of a closed cylindrical spline surface on two opposite edges of this surface, the periodicity conditions were set (the value at nodes for $j=m$ coincide with the values for $j=0)$ :

$$
\begin{aligned}
& \partial_{t}^{1} \mathbf{r}^{(i, m-1)}(0,1)=\partial_{t}^{1} \mathbf{r}^{(i, 0)}(0,0), \\
& \partial_{t t}^{2} \mathbf{r}^{(i, m-1)}(0,1)=\partial_{t t}^{2} \mathbf{r}^{(i, 0)}(0,0), i=0 . . n .
\end{aligned}
$$

Therefore, to determine the unknown quantities, we obtain following Equations (11).

$$
\begin{aligned}
& \partial_{t}^{1} \mathbf{r}_{i, 0}-\partial_{t}^{1} \mathbf{r}_{i, m}=0, i=0 . . n, \\
& 2 \partial_{t}^{1} \mathbf{r}_{i, 0}+\partial_{t}^{1} \mathbf{r}_{i, 1}+\partial_{t}^{1} \mathbf{r}_{i, m-1}+2 \partial_{t}^{1} \mathbf{r}_{i, m}= \\
& -3 M_{i, 0}+3 M_{i, 1}-3 M_{i, m-1}+3 M_{i, m} .
\end{aligned}
$$

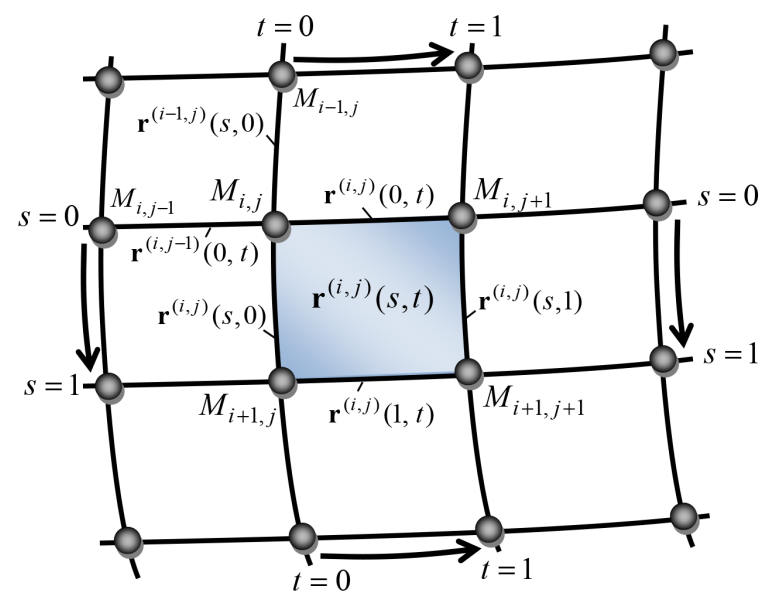

Figure 4. The layout of nodes for the element $\mathbf{r}^{(i, j)}(t, s)$ spline surface

On the other two free edges (at $i=0$ and $i=n$ ) we set the conditions for the zero derivatives of the first derivatives (12).

$$
\partial_{s}^{1} \mathbf{r}^{(0, j)}(0,0)=0, \partial_{s}^{1} \mathbf{r}^{(n-1, j)}(1,0)=0, \quad j=0 . . m,
$$

where we obtain the equation for values:

$$
\partial_{s}^{1} \mathbf{r}_{0, j}=0, \partial_{s}^{1} \mathbf{r}_{n, j}=0,=0 . . m .
$$

\section{Algorithm for calculating the Hermite interpolation surface}

The easiest way to construct a spline function of two variables is to consistently apply the algorithm for calculating the spline function of one variable (Zavyalov, Kvasov, \& Miroshnichenko, 1980; Kvass, 2006). Thus, to determine the three arrays of unknown values of the derivatives $\partial_{t}^{1} \mathbf{r}_{i, j}, \partial_{s}^{1} \mathbf{r}_{i, j}, \partial_{t s}^{2} \mathbf{r}_{i, j}$ in the points $M_{i, j}, i=0 . . n, j=0 . . m$ you need to complete three stages of calculations:

1) to calculate the values $\partial_{t}^{1} \mathbf{r}_{i, j}$, it is necessary to construct the splines of a single variable $t$, using the rows of the matrix $M_{i, j}, i=0 . . n$ (14), (15).

$$
\begin{gathered}
\mathbf{r}^{(i, j)}(0, t)=S^{T}(0) \mathbf{B} \mathbf{M}^{(i, j)} \mathbf{B}^{T} T(t)= \\
{\left[\begin{array}{llll}
\mathbf{r}_{i, j} & \mathbf{r}_{i, j+1} & \partial_{t}^{1} \mathbf{r}_{i, j} & \partial_{t}^{1} \mathbf{r}_{i, j+1}
\end{array}\right]\left[\begin{array}{cccc}
1 & 0 & 0 & 0 \\
0 & 0 & 1 & 0 \\
-3 & 3 & -2 & -1 \\
2 & -2 & 1 & 1
\end{array}\right]^{T}\left[\begin{array}{c}
1 \\
t \\
t^{2} \\
t^{3}
\end{array}\right] .}
\end{gathered}
$$

According to conditions (5) and (6), the problem of determination $\partial_{t}^{1} \mathbf{r}_{i, j}$ is reduced to solving linear threediagonal matrix Equations (15).

$$
\left[\begin{array}{cccccccc}
1 & & & & & & -1 \\
1 & 4 & 1 & & & & \\
& 1 & 4 & 1 & & & \\
& & \ldots & \ldots & \ldots & & \\
& & & 1 & 4 & 1 & \\
& & & & 1 & 4 & 1 \\
2 & 1 & & & & 1 & 2
\end{array}\right]\left[\begin{array}{ccccc}
\partial_{t}^{1} \mathbf{r}_{0,0} & \partial_{t}^{1} \mathbf{r}_{1,0} & \ldots & \partial_{t}^{1} \mathbf{r}_{n, 0} \\
\partial_{t}^{1} \mathbf{r}_{0,1} & \partial_{t}^{1} \mathbf{r}_{1,1} & \ldots & \partial_{t}^{1} \mathbf{r}_{n, 1} \\
\partial_{t}^{1} \mathbf{r}_{0,2} & \partial_{t}^{1} \mathbf{r}_{1,2} & \ldots & \partial_{t}^{1} \mathbf{r}_{n, 2} \\
\ldots & \ldots & \ldots & \ldots \\
\ldots & \ldots & \ldots & \ldots \\
\partial_{t}^{1} \mathbf{r}_{0, m-1} & \partial_{t}^{1} \mathbf{r}_{1, m-1} & \ldots & \partial_{t}^{1} \mathbf{r}_{n, m-1} \\
\partial_{t}^{1} \mathbf{r}_{0, m} & \partial_{t}^{1} \mathbf{r}_{1, m} & \ldots & \partial_{t}^{1} \mathbf{r}_{n, m}
\end{array}\right]=
$$




$$
\left[\begin{array}{ccccccc}
0 & 0 & & & & & \\
-3 & 0 & 3 & & & & \\
& -3 & 0 & 3 & & & \\
& & \ldots & \ldots & \ldots & & \\
& & & -3 & 0 & 3 & \\
& & & & -3 & 0 & 3 \\
-3 & 3 & & & & -3 & 3
\end{array}\right]\left[\begin{array}{cccc}
M_{0,0} & M_{1,0} & \ldots & M_{n, 0} \\
M_{0,1} & M_{1,1} & \ldots & M_{n, 1} \\
M_{0,2} & M_{1,2} & \ldots & M_{n, 2} \\
\ldots & \ldots & \ldots & \ldots \\
\ldots & \ldots & \ldots & \ldots \\
M_{0, m-1} & M_{1, m-1} & \ldots & M_{n, m-1} \\
M_{0, m} & M_{1, m} & \ldots & M_{n, m}
\end{array}\right] .
$$

The number of equations for each (of the three) systems corresponds to $m-1$ internal nodes of the $i$-th row of the matrix $M_{i j}$ (the first condition of continuity on each internal node) plus two boundary conditions of the extreme nodes (the first and last lines), that is, together $m+1$ equations. Given the number of rows, we have $(m+1) \times(n+1)$ equations to determine the same number of unknowns $\partial_{t}^{1} \mathbf{r}_{i, j}$.

2) to calculate the values $\partial_{s}^{1} \mathbf{r}_{i, j}$, we construct the splines from the variable $s$ on the columns of the matrix $M_{i j}, j=0 . . m$ (16), (17).

$$
\begin{gathered}
\mathbf{r}^{(i, j)}(s, 0)=S^{T}(s) \mathbf{B} \mathbf{M}^{(i, j)} \mathbf{B}^{T} T(0)= \\
{\left[\begin{array}{llll}
1 & s & s^{2} & s^{3}
\end{array}\right]\left[\begin{array}{cccc}
1 & 0 & 0 & 0 \\
0 & 0 & 1 & 0 \\
-3 & 3 & -2 & -1 \\
2 & -2 & 1 & 1
\end{array}\right]\left[\begin{array}{c}
\mathbf{r}_{i, j} \\
\mathbf{r}_{i+1, j} \\
\partial_{s}^{1} \mathbf{r}_{i, j} \\
\partial_{s}^{1} \mathbf{r}_{i+1, j}
\end{array}\right] .}
\end{gathered}
$$

According to conditions (14) and (15) for determination $\partial_{s}^{1} \mathbf{r}_{i, j}$ we have similar linear matrix equations of the form:

$$
\left[\begin{array}{cccccc}
1 & & & & \\
1 & 4 & 1 & & \\
& \ldots & \ldots & \ldots & \\
& & 1 & 4 & 1 \\
& & & & 1
\end{array}\right]\left[\begin{array}{ccccc}
\partial_{s}^{1} \mathbf{r}_{0,0} & \partial_{s}^{1} \mathbf{r}_{0,1} & \partial_{s}^{1} \mathbf{r}_{0,2} & \ldots & \partial_{s}^{1} \mathbf{r}_{0, m} \\
\partial_{s}^{1} \mathbf{r}_{1,0} & \partial_{s}^{1} \mathbf{r}_{1,1} & \partial_{s}^{1} \mathbf{r}_{1,2} & \ldots & \partial_{s}^{1} \mathbf{r}_{1, m} \\
\ldots & \ldots & \ldots & \ldots & \ldots \\
\ldots & \ldots & \ldots & \ldots & \ldots \\
\partial_{s}^{1} \mathbf{r}_{n, 0} & \partial_{s}^{1} \mathbf{r}_{n, 1} & \partial_{s}^{1} \mathbf{r}_{n, 2} & \ldots & \partial_{s}^{1} \mathbf{r}_{n, m}
\end{array}\right]=
$$

$$
\left[\begin{array}{ccccc}
0 & 0 & & & \\
-3 & 0 & 3 & & \\
& \ldots & \ldots & \ldots & \\
& & -3 & 0 & 3 \\
& & & 0 & 0
\end{array}\right]\left[\begin{array}{ccccc}
M_{0,0} & M_{0,1} & M_{0,2} & \ldots & M_{0, m} \\
M_{1,0} & M_{1,1} & M_{1,2} & \ldots & M_{1, m} \\
\ldots & \ldots & \ldots & \ldots & \ldots \\
\ldots & \ldots & \ldots & \ldots & \ldots \\
M_{n, 0} & M_{n, 1} & M_{n, 2} & \ldots & M_{n, m}
\end{array}\right]
$$

where there are $n-1$ continuity conditions in the internal nodes of $j$-th column of the matrix $M_{i j}$ plus two boundary conditions of the extreme nodes (the first and last lines), that is, together $n+1$ equations. As a result, we also obtain $(n+1) \times(m+1)$ equations for determining the same number of unknowns $\partial_{s}^{1} \mathbf{r}_{i, j}$.

3) to find the values of mixed derivatives $\partial_{s t}^{2} \mathbf{r}_{i, j}$, there are two options for constructing a spline of one variable - by rows of the matrix $\partial_{s}^{1} \mathbf{r}_{i, j}$ or by columns of the matrix $\partial_{t}^{1} \mathbf{r}_{i, j}$, obtained in the previous stages of the calculation, requiring continuity of third $\partial_{s t t}^{3} \mathbf{r}^{(i, j)}(s, t)$ or $\partial_{t s s}^{3} \mathbf{r}^{(i, j)}(s, t)$ derivatives and satisfying the corresponding boundary conditions. In essence, the conditions of continuity of third derivatives mean the continuous rate of change of the second derivatives in the direction, determined by the parameter $s$ or $t$. In order to compare the results, we will implement both versions of the construction. Here, for example, we present a system of equations for calculating values $\partial_{s t}^{2} \mathbf{r}_{i, j}$ as a result of constructing splines from a variable $t$ in rows of a matrix $\partial_{s}^{1} \mathbf{r}_{i, j}$, obtained in the second stage. To do this, we write the continuity conditions for third derivatives $\partial_{s t t}^{3} \mathbf{r}^{(i, j)}(s, t)$ in the internal nodes:

$$
\partial_{s t t}^{3} \mathbf{r}_{i, j}(0,1)=\partial_{s t t}^{3} \mathbf{r}_{i, j}(0,0), i=0 . . n, j=1 . . m-1
$$

or by calculating the derivatives of expressions (2) and moving the unknowns to the left, we will get:

$$
\begin{aligned}
& \partial_{s t}^{2} \mathbf{r}_{i, j-1}+4 \partial_{s t}^{2} \mathbf{r}_{i, j}+\partial_{s t}^{2} \mathbf{r}_{i, j+1}=-3 \partial_{s}^{1} \mathbf{r}_{i, j-1}+3 \partial_{s}^{1} \mathbf{r}_{i, j+1}, \\
& i=0 . . n, j=1 . . m-1 .
\end{aligned}
$$

In the outermost nodes, we define the conditions for periodicity (surface closure):

$$
\begin{aligned}
& \partial_{s t}^{2} \mathbf{r}^{(i, m-1)}(0,1)=\partial_{s t}^{2} \mathbf{r}^{(i, 0)}(0,0), \\
& \partial_{s t t}^{3} \mathbf{r}^{(i, m-1)}(0,1)=\partial_{s t t}^{3} \mathbf{r}^{(i, 0)}(0,0), i=0 . . n,
\end{aligned}
$$

that is, to determine the unknown quantities, we obtain the corresponding equations:

$$
\begin{aligned}
& \partial_{s t}^{2} \mathbf{r}_{i, 0}-\partial_{s t}^{2} \mathbf{r}_{i, m}=0, i=0 . . n, \\
& 2 \partial_{s t}^{2} \mathbf{r}_{i, 0}+\partial_{s t}^{2} \mathbf{r}_{i, 1}+\partial_{s t}^{2} \mathbf{r}_{i, m-1}+2 \partial_{s t}^{2} \mathbf{r}_{i, m}= \\
& -3 \partial_{s}^{1} \mathbf{r}_{i, 0}+3 \partial_{s}^{1} \mathbf{r}_{i, 1}-3 \partial_{s}^{1} \mathbf{r}_{i, m-1}+3 \partial_{s}^{1} \mathbf{r}_{i, m} .
\end{aligned}
$$

So, according to conditions (15) and (16), the matrix equations for the definition $\partial_{s}^{1} \mathbf{r}_{i, j}$ will be:

$$
\begin{aligned}
& {\left[\begin{array}{ccccccc}
1 & & & & & & -1 \\
1 & 4 & 1 & & & & \\
& 1 & 4 & 1 & & & \\
& & \cdots & \cdots & \ldots & & \\
& & & \cdots & \ldots & \ldots & \\
& & & & 1 & 4 & 1 \\
2 & 1 & & & & 1 & 2
\end{array}\right]\left[\begin{array}{ccccc}
\partial_{s t}^{2} \mathbf{r}_{0,0} & \partial_{s t}^{2} \mathbf{r}_{1,0} & \ldots & \partial_{s t}^{2} \mathbf{r}_{n, 0} \\
\partial_{s t}^{2} \mathbf{r}_{0,1} & \partial_{s t}^{2} \mathbf{r}_{1,1} & \ldots & \partial_{s t}^{2} \mathbf{r}_{n, 1} \\
\partial_{s t}^{2} \mathbf{r}_{0,2} & \partial_{s t}^{2} \mathbf{r}_{1,2} & \ldots & \partial_{s t}^{2} \mathbf{r}_{n, 2} \\
\ldots & \ldots & \ldots & \ldots \\
\ldots & \ldots & \ldots & \ldots \\
\partial_{s t}^{2} \mathbf{r}_{0, m-1} & \partial_{s t}^{2} \mathbf{r}_{1, m-1} & \ldots & \partial_{s t}^{2} \mathbf{r}_{n, m-1} \\
\partial_{s t}^{2} \mathbf{r}_{0, m} & \partial_{s t}^{2} \mathbf{r}_{1, m} & \ldots & \partial_{s t}^{2} \mathbf{r}_{n, m}
\end{array}\right]=} \\
& {\left[\begin{array}{cccccccc}
0 & 0 & & & & & \\
-3 & 0 & 3 & & & & \\
& -3 & 0 & 3 & & & \\
& & \ldots & \ldots & \ldots & & \\
& & & \ldots & \ldots & \ldots & \\
& & & & -3 & 0 & 3 \\
-3 & 3 & & & & -3 & 3
\end{array}\right]\left[\begin{array}{ccccc}
\partial_{s}^{1} \mathbf{r}_{0,0} & \partial_{s}^{1} \mathbf{r}_{1,0} & \ldots & \partial_{s}^{1} \mathbf{r}_{n, 0} \\
\partial_{s}^{1} \mathbf{r}_{0,1} & \partial_{s}^{1} \mathbf{r}_{1,1} & \ldots & \partial_{s}^{1} \mathbf{r}_{n, 1} \\
\partial_{s}^{1} \mathbf{r}_{0,2} & \partial_{s}^{1} \mathbf{r}_{1,2} & \ldots & \partial_{s}^{1} \mathbf{r}_{n, 2} \\
\ldots & & \ldots & \ldots & \ldots \\
\ldots & \ldots & \ldots & \ldots \\
\partial_{s}^{1} \mathbf{r}_{0, m-1} & \partial_{s}^{1} \mathbf{r}_{1, m-1} & \ldots & \partial_{s}^{1} \mathbf{r}_{n, m-1} \\
\partial_{s}^{1} \mathbf{r}_{0, m} & \partial_{s}^{1} \mathbf{r}_{1, m} & \ldots & \partial_{s}^{1} \mathbf{r}_{n, m}
\end{array}\right] .}
\end{aligned}
$$


It should be noted, that the value of mixed derivatives $\partial_{s t}^{2} \mathbf{r}_{i, j}$, obtained from other conditions, will generally differ.

Finally, using computed arrays of partial derivatives $\partial_{t}^{1} \mathbf{r}_{i, j}, \partial_{s}^{1} \mathbf{r}_{i, j}, \partial_{t s}^{2} \mathbf{r}_{i, j}$, at given points $M_{i j}$, and by using the formulas (2), we can build fragments of the Hermite interpolation bicubic surface.

\section{Results of the research}

Using the created algorithm we obtained matrices containing information about the coordinates of four points on the corners of the surface and partial derivatives at these points. For example, we give the value for the fragment $i=3, j=5$ :

$M^{(3.5)}=\left[\begin{array}{l}{\left[\begin{array}{l}81.0270 \\ 131.069 \\ 110.045\end{array}\right]\left[\begin{array}{l}76.0740 \\ 137.3000 \\ 110.032\end{array}\right]\left[\begin{array}{l}-4.3554 \\ 6.6038 \\ -0.0083\end{array}\right]\left[\begin{array}{l}-4.382 \\ 6.6148 \\ 0.0079\end{array}\right]} \\ {\left[\begin{array}{l}80.9800 \\ 131.062 \\ 107.567\end{array}\right]\left[\begin{array}{l}76.052 \\ 137.303 \\ 107.581\end{array}\right]\left[\begin{array}{l}-5.5796 \\ 5.732 \\ 0.0114\end{array}\right]\left[\begin{array}{l}-5.5376 \\ 5.7193 \\ -0.0005\end{array}\right]} \\ {\left[\begin{array}{l}-0.0263 \\ -0.0042 \\ -2.7524\end{array}\right]\left[\begin{array}{l}-0.0190 \\ 0.0023 \\ -2.7199\end{array}\right]\left[\begin{array}{l}-0.030 \\ 0.0065 \\ 0.0269\end{array}\right]\left[\begin{array}{l}-0.0077 \\ -0.0047 \\ -0.0008\end{array}\right]} \\ {\left[\begin{array}{l}-0.0147 \\ -0.002 \\ -2.1741\end{array}\right]\left[\begin{array}{l}-0.0044 \\ 0.0006 \\ -2.1540\end{array}\right]\left[\begin{array}{l}0.0278 \\ -0.0089 \\ 0.0006\end{array}\right]\left[\begin{array}{l}0.0105 \\ 0.0023 \\ -0.0081\end{array}\right]}\end{array}\right.$

Using (2) and (23), a bicubic spline was found, which defines the surface for $i=3, j=5$ :

$$
\begin{aligned}
& r^{(3.5)}(s, t)= \\
& {\left[\begin{array}{l}
4.825177 s^{3} t^{3}-7.303916 s^{3} t^{2}+2.446339 s^{3} t^{1}+ \\
0.59764 s^{3} t^{0}+\ldots+81.027 \\
3.551345 s^{3} t^{3}-5.303258 s^{3} t^{2}+1.741183 s^{3} t^{1}+ \\
0.007636 s^{3} t^{0}+\ldots+131.069 \\
0.001256 s^{3} t^{3}+0.011826 s^{3} t^{2}-0.011934 s^{3} t^{1}+ \\
0.029455 s^{3} t^{0}+\ldots+110.045
\end{array}\right] .}
\end{aligned}
$$

Where the first element of the vector contains a bicubic polynomial of 16 members, which defines $X(s, t)$, the second $-Y(s, t)$ and $Z(s, t)$ - the third.

By using the obtained function, a fragment of the surface was constructed.

The surface of the VST of the LODS "Brody" company (Figure 5a) was obtained by stitching of 240 surfaces (Figure $5 b$ ). These surfaces will constructed, using the proposed algorithm, based on bicubic spline interpolation.

The Figure $5 \mathrm{c}$ clearly shows the presence of torsion and bending of the plot. The use of a full bicubic spline for a)

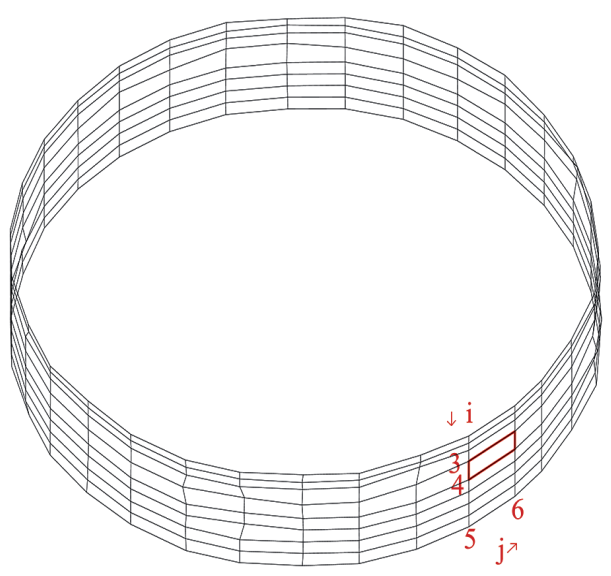

b)

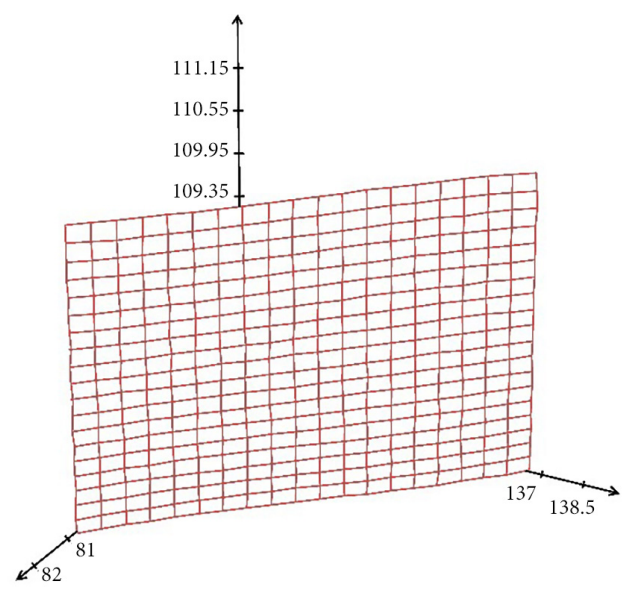

c)

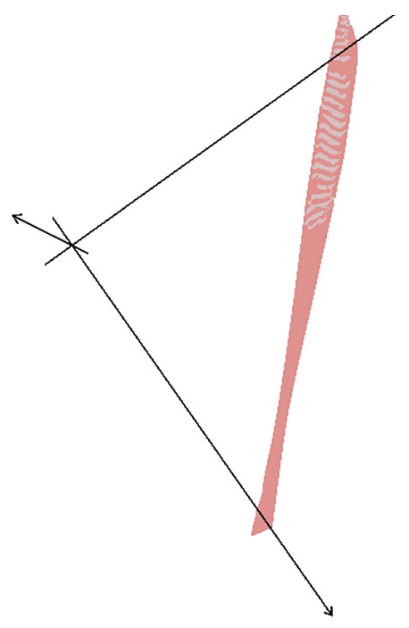

Figure 5. a) 3D visualization of the surface of the VST 75.000 $\mathrm{m}^{3}$ LODS "Brody"; b) a fragment of the interpolation bicubic Hermite surface for the region $i=3 j=5$; c) the top view

the construction of surfaces ensures the presence of these characteristics. The information content of the obtained results in the form of images allows to be used them for a detailed analysis of the technical condition of tanks.

\section{Scientific novelty and practical significance}

Currently, there are several software systems, such as MathCAD, Surfer, MatLab (Rovenski, 2010), which allow 
you to build surfaces and find the value of spatial coordinates in the required locations of these surfaces, but because of the closeness of the program code, users do not know the principles of the algorithms used. Also, obtaining a complete bicubic polynomial, if it was used for construction, is impossible. Therefore, an important aspect of using the proposed algorithm is precisely the possibility of obtaining a complete bicubic polynomial of a spatial spline, describing a 3D surface, because it is necessary to work with functions for solving many engineering problems.

On the basis of the conducted research, it was established that the created algorithm can be used for the practical implementation of geodetic surveys using the example of a vertical cylindrical steel welded tank with a nominal capacity of $75.000 \mathrm{~m}^{3}$ with a floating roof and a double wall of the LODS "Brody" company.

\section{Conclusions}

The method, proposed by the authors, for processing the results of geodetic surveys allows to improve the accuracy, information content and quality of determining the geometric parameters of vertical steel tanks.

An algorithm for processing data of instrumental geodetic observations has been created allows to creating 3D surfaces of tanks, using the complete bicubic polynomial.

The clarity of the obtained results will allow to improving the quality of the technical condition assessment, increase the operational reliability and extend the service life of the tanks.

The resulting spline functions allow to solve a number of engineering problems, for example, determining the stressed-deformed state of steel tanks.

Future studies are planned to be devoted to the use of bicubic spline interpolation to determine the geometric parameters of vertical steel tanks.

\section{References}

Alberg, J., Nilson, E., \& Walsh, J. (1972). Spline theory and its applications. Moscow: Mir.

Burak, K., Kovtun, V., Levitskiy, R., \& Nychvyd, M. (2014). Investagation the characteristic and the accuracy of DTM construction using bicubic spline interpolation. Modern Achievements in Geodetic Science and Industry, 2, 32-36. Retrieved from http://nbuv.gov.ua/j-pdf/sdgn_2014_2_9.pdf

Burak, K., Kovtun, V., \& Levitskiy, R. (2015). Determination of the density of a bicubic spline interpolation regular grid when creating DEM. Modern Achievements in Geodetic Science and Industry, 1, 133-139. Retrieved from http://nbuv.gov. ua/UJRN/sdgn_2015_1_34

DSTU-NBA.3.1-10:2008 FAT. (2008). Guidelines for the technical diagnosis of vertical steel tanks. Retrieved from https://issuu. com/igel99778/docs/dstu-n_b_a_3_1-10_2008_instrukciya

GOST 8.570-2000. (2000). State system for ensuring uniformity of measurements. Steel vertical cylindrical tanks. Method of verification. Retrieved from https://internet-law.ru/gosts/ gost/6533/

Komissarov, Seredovich, A. V., \& Ivanova, A. V. (2006). Method of determining the geometric characteristics of steel cylindrical tanks with the introduction of laser scanning. Interexpo Geo-Siberia, 1, 221-225.

Kvass, B. I. (2006). Methods of isogeometric approximation by splines. Moscow: FIZMATLIT.

Larichev, V. A., Lesonen, D. N., Maksimov, G. A., Podgyachev, E. V., \& Derov, A. V. (2010). About the approach to three-dimensional mathematical modeling of a complex geological environment with gaps for visualization and solving direct and inverse problems of geophysics. Scientific Visualization, 2(2), 20-33.

Rovenski, V. (2010). Modeling of curves and surfaces with MAT$L A B$. New York: Springer-Verlag. https://doi.org/10.1007/978-0-387-71278-9

Samoilenko, A. N., \& Zaets, V. V. (2007). Raising the accuracy in determining the geometrical parameters and calibration of vertical cylindrical vessels on checking. Measurement Techniques, 50(3), 266-271. https://doi.org/10.1007/s11018-007-0059-6

Seredovich, A. V., \& Ivanov, A. V. (2006). Development of methods for determining the geometric parameters of the RVS based on laser scanning data. Interexpo Geo-Siberia, 2, 160164.

Shikin, E. V., \& Plis, L. I. (1996). Curved surfaces on the computer screen. Spline user guide. Moscow: DIALOG-MEPI.

SUC 60.3.31570412:2010. (2010). The main oil pipelines. Oil pumping station, sea terminals, technical inspection, expert inspection of process equipment and pipelines. Methods and techniques.

Tarasenko, M. I., \& Tishchenko, A. (2009). Method of determining the technical parameters of electronic tachometers when operating in non-reflective mode. Geodesy, Cartography and Aerial Photography: an Interdepartmental Scientific and Technical Collection, 72, 54-61.

Trevogo, I. S., Ilkiv, E. U., \& Kukhtar, D. V. (2013). Optimization of the use of film reflectors for monitoring deformations of engineering structures. Geodesy, Cartography and Aerial Photography: Interdepartmental Scientific and Technical Collection, 78, 146-148.

Zavyalov, Yu. S., Kvasov, B. I., \& Miroshnichenko, V. L. (1980). Methods of spline functions. Moscow: Science. 